\title{
The Finding of Airport Security's Bottleneck
}

\author{
Xiaochen Ren
}

North China Electric Power University, Baoding 071003, China.

18730265912@163.com

\section{Keyword: Generalized; Stochastic Petri net; Markov chain; Security process}

\begin{abstract}
This paper establishes security process optimization model based on GSPN and use average token to make performance analysis of each step in security process to identity bottlenecks and put forward some optimization suggestions.

Based on the theory of Petri Net, we divide the whole security process into several steps and set up the security process optimization model. Under the premise of validating the structural reliability of the model, we analyze the tangible state and vanishing state of the GSPN and transform the generalized stochastic Petri net into the isomorphic Markov chain to get the probability of each state and get the average token. Thus, we find that the bottlenecks in the security process are ID check and luggage collection.
\end{abstract}

\section{Introduction}

In order to ensure the safety of the airport and personnel, security check at the terminal has become the key process of flying experience. As the aircraft isincreasingly becoming a convenient way for people to travel long distances, the amount of air traffic has a substantial increase and airport passenger throughput also increases significantly. However, airlines have a vested interest in maintaining a positive flying experience for passengers by minimizing the time they spend waiting in line at a security checkpoint and waiting for their flight. During 2016, the U.S. Transportation Security Agency (TSA) came under sharp criticism for extremely long lines due to the long waiting time. Although TSA invested in several modifications to their checkpoint equipment and procedures and increased staffing in the more highly congested, these optimization plans cause a lot of expenditure to maintain the passenger throughput.

\section{Security Process Decomposition}

Through the description and analysis of the security process in the background, we divide the whole security process into the following steps:

The passengers arrived at the terminal wait in a queue until a security officer can inspect their identification and boarding documents

The passengers prepare all of their belongings for X-ray screening and meanwhile the passenger process through either a millimeter wave scanner or metal detector.

All of the belongings, including the bins containing the aforementioned items, are moved by conveyor belt through an X-ray machine, where some items are flagged for additional search or screening by a security officer. Passengers that fail this step receive a pat-down inspection by a security officer.

After inspection, the passengers collect their luggage and exit.

\section{Model Establishment}

Through the decomposition of the security process, we regard the whole process as a collection of discrete events. The security process has the characteristic of synchronization and concurrency. Therefore, we introduce the time parameter to analyze the time behavior of the model and establish the security process optimization model based on generalized stochastic Petri net.

In the model constriction, we utilize place to express the state before the security process 
execution, utilize transition to express activities in various executions in the security process and utilize connections to express the location of service objects during the security process. Eventually, we get the security process optimization model based on generalized stochastic Petri net as following:

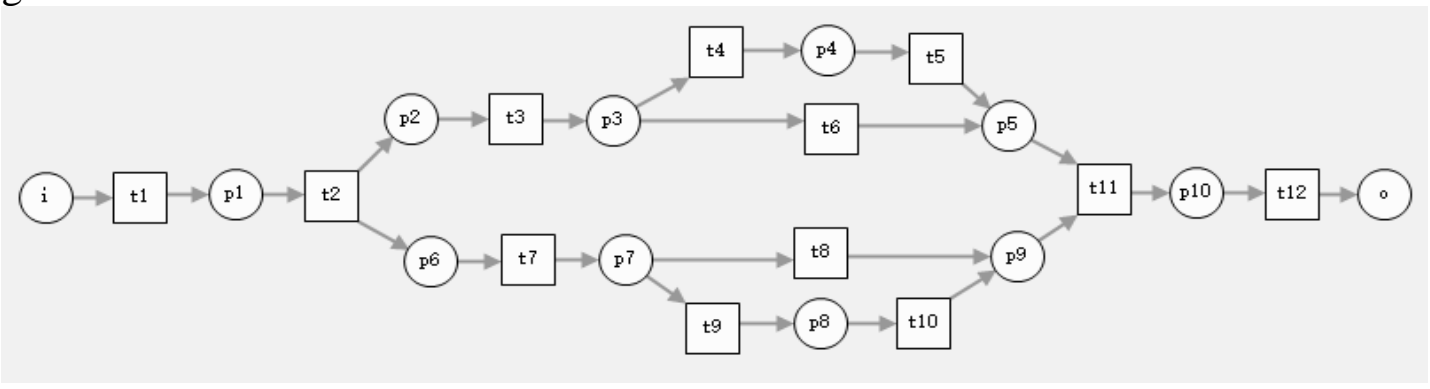

Figure 1. The security process model based on GSPN

The definition of symbols are interpreted as following:

Table 1 the definition of place

\begin{tabular}{l|l}
\hline Place & Definition \\
\hline$i$ & Prepare for ID check \\
\hline$p_{1}$ & Passengers after ID check \\
\hline$p_{2}$ & Luggage to be X-ray scanned \\
\hline$p_{3}$ & Luggage after X-ray scanner \\
\hline$p_{4}$ & Luggage need to have open-package inspection \\
\hline$p_{5}$ & Luggage after open-package inspection \\
\hline$p_{6}$ & Passengers to be millimeter wave scanned \\
\hline$p_{7}$ & Passengers after millimeter wave scanner \\
\hline$p_{8}$ & Passengers need to have personal examination \\
\hline$p_{9}$ & Passengers after personal examination \\
\hline$p_{10}$ & Passengers intend to collect luggage \\
\hline$o$ & Passengers who have collected their luggage \\
\hline
\end{tabular}

Table 2 The definition of transitions

\begin{tabular}{l|l}
\hline Transition & Definitions \\
\hline$t_{1}$ & ID check \\
\hline$t_{2}$ & Prepare for security check \\
\hline$t_{3}$ & X-ray scan \\
\hline$t_{4}$ & Passenger's luggage with suspicious objects \\
\hline$t_{5}$ & Open -package check \\
\hline$t_{6}$ & Passenger's luggage without suspicious objects \\
\hline$t_{7}$ & Go through either a millimeter wave scanner or metal detector \\
\hline$t_{8}$ & The millimeter wave scanner alarms \\
\hline$t_{9}$ & The millimeter wave scanner doesn't alarm \\
\hline$t_{10}$ & Personal check \\
\hline$t_{11}$ & Transmission of luggage on the belt \\
\hline$t_{12}$ & Luggage collection \\
\hline
\end{tabular}




\section{Model Solution}

Data Interpretation. Dataset, each column of data, is independent of others as several groups of people were coming through the screening process.

Before the simulation of the model, we process the data by the following steps:

Find the mean value and variance of the consumption time of each transition.

We assume that data with large deviation degree is invalid and get more efficient data.

\section{Simulation Results}

Using the processed data, we establish the security process optimization model based on GSPN and evaluate its performance. we calculate the density matrix X:

$$
\left\{\begin{array}{l}
-5.6604 x_{1}+\lambda x_{11}=0 \\
5.6604 x_{1}-24.0688 x_{2}=0 \\
2.4631 x_{2}-6.1216 x_{3}=0 \\
16.4841 x_{2}+x_{3}-5.1216 x_{4}=0 \\
0.2561 x_{2}-21,9472 x_{5}=0 \\
4.8655 x_{2}+3 x_{5}-18.9472 x_{6}=0 \\
0.2561 x_{3}+2.4631 x_{5}-4 x_{7}=0 \\
0.9473 x_{4}+16.4841 x_{5}+x_{7}-3 x_{8}=0 \\
4.8655 x_{3}+2.4631 x_{6}+3 x_{7}-x_{9}=0 \\
4.8655 x_{4}+164841 x_{6}+3 x_{8}+x_{9}-2.0964 x_{10}=0 \\
2.0964 x_{10}-\lambda x_{11}=0 \\
\sum x_{i}=1
\end{array}\right.
$$

Solving the function $X \cdot Q=0$, we get the probability distribution and obtain the average token of each place of the security process model based on GSPN.We can get the result that places $\left(i, p_{10}\right)$ are the bottlenecks of the security process. $i$ represents the process of ID check and ${ }^{p_{10}}$ represents the process that passengers intend to collect luggage.

\section{Summary}

Based on GSPN and the theory of Petri Net,this papaer establishes a security process optimization model.Then we calculate the density matrix and get the result is that the bottlenecks of the security process are represents the process of ID check and represents the process that passengers intend to collect luggage. If we want to increase the efficiency of security, we need to improve the speed of this two processes.

\section{Reference}

[1] Jianjun Ma. Virtual queuing in the application of civil aviation security system [J]. Science Technology and Innovation, 2016, (19) : 34 and 35.

[2] Guoyang Jia. The Research about Terminal Passenger Security Service Process Optimization based on LCIOWF [D]. Harbin Institute of Technology 2015.

[3] Xinxin Jiang, Hang Zhou, Bingqing Cai. The Study of Terminal Security Layout and Process Optimization [J]. Aerospace Computing Technology, 2015, (3) : 25-29 + 34.

[4] Jinsong Zhang.Modeling and Simulation Study of Passenger Departure Process in Hub Airport based on Petri Net [D]. China Civil Aviation Flight Institute, 2015.

[5] Junjie Zeng. Settings and Optimization of Airport Security [J]. Journal of Knowledge Economy, 2009, (12) : 173-174. 